\title{
On the Characteristics of the Scales of the Atmospheric Motions
}

\author{
By Tu-cheng Yeh and Mai-tsun Li \\ Institute of Atmospheric Physics, Academia Sinica \\ (Manuscript received 12 September 1981)
}

\begin{abstract}
It is shown in this paper that the development of most of the atmospheric weather systems, large-scale or small-scale, consists of three stages, namely the adjustment stage (called geostrophic adjustment for large-scale motions), the developing stage and the quasisteady stage. There are three time scales corresponding to these three stages of development, the time scale for the adjustment stage being shortest, that for the developing stage being much longer and that for quasi-steady stage being longest. The physical processes in the development of these three stages are related to the evolution of the potential vorticity field.

It is further shown in this paper that similar to the scale effect in large-scale motion with a critical scale $L_{0}=c / f$, there is also a critical scale $L_{0}^{\prime}=c / \Omega$ for meso-scale motion, where $c$ is the speed of gravity wave; $f$, the Coriolis parameter and $\Omega$, the characteristic value of absolute vorticity of the -wind. Under certain assumptions, a critical scale $L_{0}^{\prime \prime}=\left[u^{2} / \frac{g}{\rho} \frac{\partial \rho}{\partial z}\right]^{1 / 2}$ can also found for motions of cumulus scale, where $u$ and $\rho$ are the characteristic velocity and density of the atmosphere respectively. The roles of $L_{0}^{\prime}$ and $L_{0}^{\prime \prime}$ in meso-scale and cumulus-scale motions are similar to that of $L_{0}$ for large-scale motions.
\end{abstract}

\section{Introduction}

The large-scale motions in the atmosphere are known to proceed under quasi-balanced forces, the quasi-balance between pressure gradient force and Coriolis force to make the motion quasigeostrophic. When this quasi-geostrophic state breaks down, there will be a process called geostrophic adjustment to bring the motion back to quasi-geostrophy. After the adjustment the motion enters into its evolutional state (Zeng, 1963). Yeh and $\mathrm{Li}$ (1965) showed that in meso- and small-scale motions there also exists a state of quasi-balanced forces. When this quasi-balance breaks down, there will also be a process to adjust the motion to quasi-balance in a very short time and then to bring the motion to evolutional state. Because the time scale of the adjustment of motions of all scales is too short, we usually can not observe this stage in the atmosphere. Synoptically what we can observe in real atmosphere is usually the evolutional stage undergoing in quasi-balanced state of forces. It is also usually observed in atmosphere that the weather systems, large or small, have two stages of development. Firstly the weather systems grow to their mature stage very rapidly and then maintain a quasi-steady state in fairly long time. For instances, the low- or high-index circulation pattern may persist several weeks (usually more than two weeks), but the transitions from high- to low-index pattern or vice versa only take a few days. The formation of a blocking high (or cut-off low) needs only two or three days, but it may maintain its intensity in quasi-steady state for more than two weeks. A low vortex usually develops within 24 hours to its mature stage and it can live several days long in almost steady condition. A convective cloud usually develops to its mature state in 10 minutes but it may last 1 to 2 hours. From these observational facts we may conclude that the evolutional stage can be divided into two sub-stages, the developing stage and quasi-steady stage. Thus together with the adjustment stage, a weather system in the atmosphere, large or small, usually possesses three stages in its development, and each stage possesses its own characteristic time scale. The problem of three time-scales was first discussed by Yeh and $\mathrm{Li}$ (1980) and later by 
Chao (1980) and others. In the present paper we shall simulate numerically the existence of the three time scales of the atmospheric motions.

For large-scale motions (adiabatic and frictionless) there is a potential vorticity equation and from this potential vorticity equation it is easy to show that there exists an effect of space-scale on the process of geostrophic adjustment. For scales larger than Rossby's radius of deformation $L_{c}=C / f, C$ and $f$ being the gravity wave speed and the Coriolis parameter respectively, the pressure field will play a predominant role in the adjustment process, while for motions of scale $L<L_{c}$, the vortical wind field will play a predominant role (Yeh, 1957; Zeng, 1963; Chen, 1963). How are about the motions of small scale? Is there also certain critical space-scale which plays the same role as $L_{c}$ in large-scale motions? Our answer is yes. In our present paper we shall derive the potential vorticity equations for meso- and small-scale motions and then from these equations we shall find for these motions the critical space-scales which will play the same role as $L_{c}$ in large-scale motions.

\section{The numerical simulations of the three time scales}

To illustrate numerically the existence of three time scales in the development of a weather system, we use the following simple barotropic and axially symmetric model

$$
\begin{aligned}
& \frac{\partial V_{r}}{\partial t}+V_{r} \frac{\partial V_{r}}{\partial r}-\frac{V_{\theta}^{2}}{r}-f V_{\theta}=-\frac{\partial \phi}{\partial r} \\
& \frac{\partial V_{\theta}}{\partial t}+V_{r} \frac{\partial V_{\theta}}{\partial r}+\frac{V_{\theta} V_{r}}{r}+f V_{r}=0 \\
& \frac{\partial \phi}{\partial t}+V_{r} \frac{\partial \phi}{\partial r}+\phi\left(\frac{\partial V_{r}}{\partial r}+\frac{V_{r}}{r}\right)=0
\end{aligned}
$$

Here $V_{r}$ and $V_{\theta}$ are respectively radial and tangential velocity and $\phi$ is the geopotential height $g h$. Equations (1) may be rewritten as

$$
\begin{aligned}
& {\left[\frac{\partial}{\partial t}+\left(V_{r}+C\right) \frac{\partial}{\partial r}\right]\left(V_{r}+2 C\right)} \\
& =V_{\theta}\left(f+\frac{V_{\theta}}{r}\right)-C \frac{V_{r}}{r} \\
& {\left[\frac{\partial}{\partial t}+\left(V_{r}-C\right) \frac{\partial}{\partial r}\right]\left(V_{r}-2 C\right)} \\
& =V_{\theta}\left(f+\frac{V_{\theta}}{r}\right)+C \frac{V_{r}}{r} \\
& {\left[\frac{\partial}{\partial t}+V_{r} \frac{\partial}{\partial r}\right] V_{\theta}=-V_{r}\left(f+\frac{V_{\theta}}{r}\right)}
\end{aligned}
$$

Where $C^{2}=\phi$. Equations (2) show that the motion consists of three waves of wave speeds $V_{r}+C, V_{r}-C$ and $V_{r}$ respectively. The first two are gravity wave speeds and the third one is the current speed. Corresponding to these three wave speeds are three characteristic lines,

$$
\frac{d r}{d t}=V_{r}+C, \frac{d r}{d t}=V_{r}-C, \frac{d r}{d t}=V_{r} .
$$

Equations (2) may be solved by method of characteristic lines. The initial conditions used are two sets:
(1) $C(r, 0)=$ constant, $V_{\theta}=V_{r}=f(r, 0)$
(2) $C(r, 0)=$ constant,

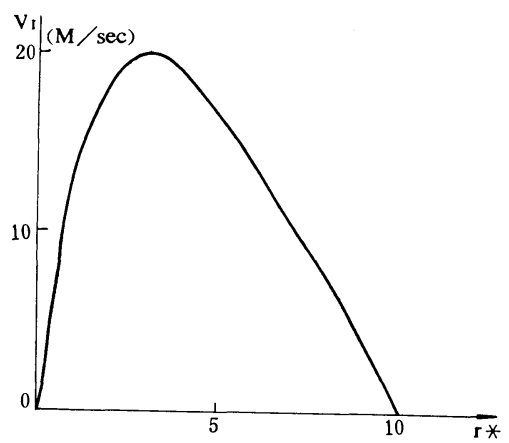

Fig. 1 The initial distribution of $V_{r}\left(r^{*}, o\right), r^{*}$ is nondimensional quantities.

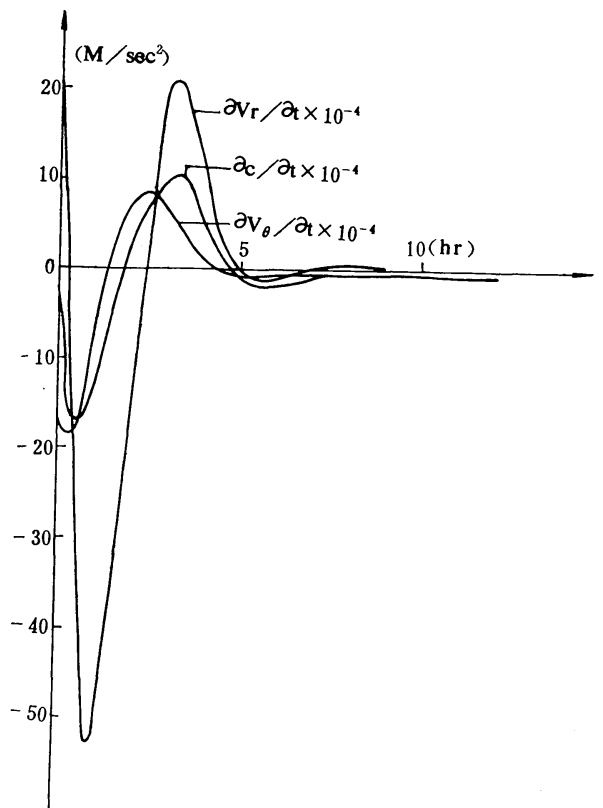

Fig. 2 The time evolution of $\partial V_{r} / \partial t, \partial V_{\theta} / \partial t$, $\partial c / \partial t$, at the $r=500 \mathrm{~km}$. 


$$
V_{\theta}(r, 0)=-V_{r}(r, 0)=f(r, 0)
$$

The first initial state is a cyclonic vortex and the second one is an anticyclonic vortex. The field of initial divergence is the same for both cases. The initial velocity distribution $f(r, O)$ is given in figure 1 , which is drawn for a non-dimensional quantity $r^{*} . r^{*}=10$ corresponds to $r=10 \mathrm{~km}$ for small-scale motion and corresponds to $r=$ $1 \mathrm{c} 0 \mathrm{~km}$ and $r=1,000 \mathrm{~km}$ for meso- and largescale motions respectively. It is seen that the initial conditions for both cases are in state of highly non-balanced forces because of uniform pressure field $[C(r, 0)=$ constant $]$. Figure 2 is the results of the computed variations with time of $\partial V_{r} / \partial t, \partial V_{\theta} / \partial t$ and $\partial c / \partial t$ at the point $r=500 \mathrm{~km}$ for an initial disturbance of $1,000 \mathrm{~km}$ in size. It is seen that within the first two to three hours, $\partial V_{\theta} / \partial t, \partial V_{r} / \partial t$ and $\partial c / \partial t$ vary very violently. After this time the variations evidently slow down and after about 10 hours, all the three quantities $\partial V_{r} / \partial t, \partial V_{\theta} / \partial t$ and $\partial c / \partial t$ almost approach to nil. This computation shows that the motion undergoes three stages. The first stage corresponds to the stage of adjustment; the second stage corresponds to the developing stage and the third stage is evidently the quasi-steady state.

The characteristics of three time stages may also be shown in the variations of the space distributions of $V_{r}, V_{\theta}$ and $C$ at different times (figure $3 \mathrm{a}, 3 \mathrm{~b}$ and $3 \mathrm{c}$ ). From these figures we may see that within first two hours the distributions of the velocity field and pressure field (c) show violent variations. Seen from figure 3a,

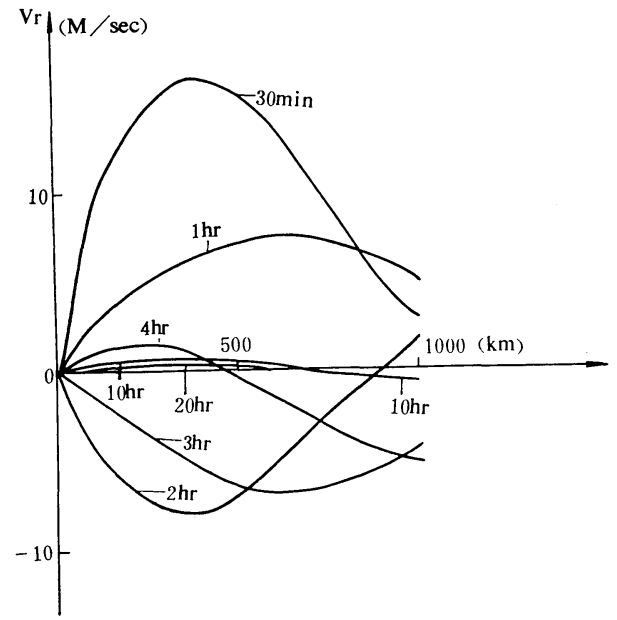

a) The radial distributions of $V_{r}$. the maximum of $V_{r}$ drops from its initial value of $20 \mathrm{~m} / \mathrm{s}$ to about $7 \mathrm{~m} / \mathrm{s}$ at the time of one hour and $V_{r}$ reverses its sign at $t=2 \mathrm{hrs}$, with an absolute maximum value of about $8 \mathrm{~m} / \mathrm{s}$. Thus within the first two hours $V_{r}$ changes about $28 \mathrm{~m} / \mathrm{s}$. From $t=2 \mathrm{hrs}$ to $t=4 \mathrm{hrs}$ the maximum value of $V_{r}$ (absolute magnitude) changes about $10 \mathrm{~m} / \mathrm{s}$ and from $t=4 \mathrm{hrs}$ to $t=10 \mathrm{hrs}$ it changes about $1.5 \mathrm{~m} / \mathrm{s}$. From $t=10 \mathrm{hrs}$ on, $V_{r}$ changes very little. Similarly the maximum value of $V_{\theta}$ (figure $3 \mathrm{~b}$ ) within the first hour drops $8.5 \mathrm{~m} / \mathrm{s}$ from its initial value of $20 \mathrm{~m} / \mathrm{s}$, from 1 hour to

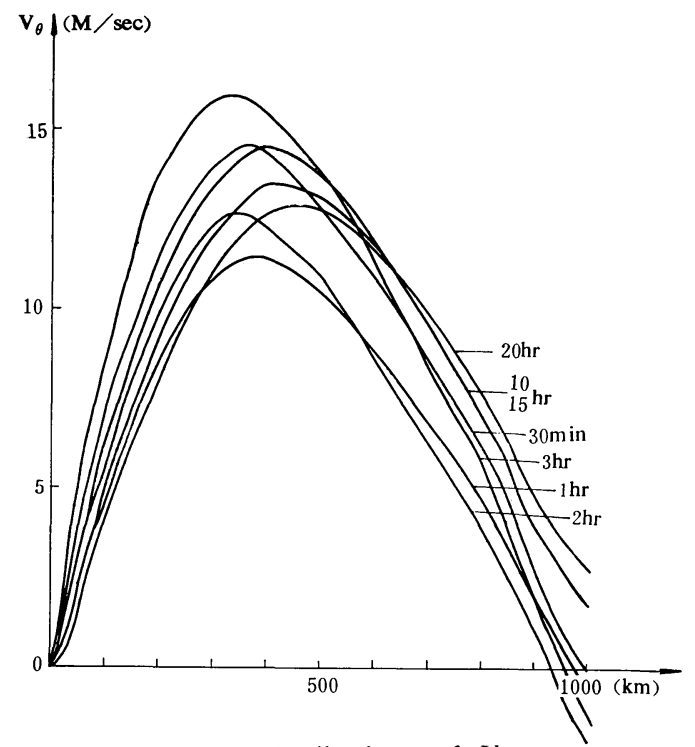

b) The radial distributions of $V_{\theta}$.

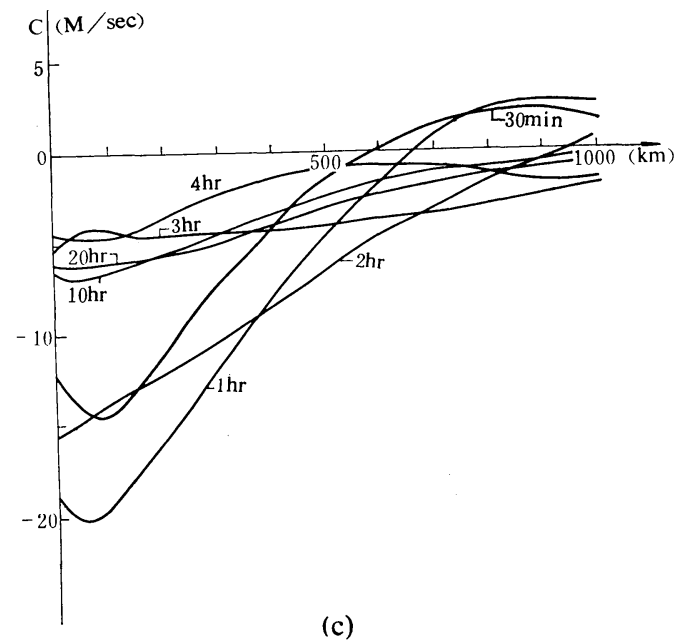

c) The radial distributions of $C$ (deviation from the initial).

Fig. 3 The radial distributions of the $V_{r}, V_{e}$ and $C$ of the large scale motion at different times. 
3 hours it rises from $11.5 \mathrm{~m} / \mathrm{s}$ to about $16.5 \mathrm{~m} / \mathrm{s}$ (about $2.5 \mathrm{~m} / \mathrm{sec} / \mathrm{hr}$ ) and from $10 \mathrm{hr}$ to $20 \mathrm{hr}$ it decreases about $2 \mathrm{~m} / \mathrm{s}$ (about $0.5 \mathrm{~m} / \mathrm{sec} / \mathrm{hr}$ ). For $C$ (figure $3 \mathrm{c}$, the distribution of which may be looked as a pressure profile), within the first hour it forms a very deep vortex with its central value of about $-20 \mathrm{~m} / \mathrm{s}$; from $t=2 \mathrm{hr}$ to about $t=10 \mathrm{hr}$ this vortex reaches about its final state and hereafter it enters in its quasi-steady state. From this computation the characteristics of three time scales in the development of a disturbance of space-scale $1,000 \mathrm{~km}$ starting from highly non-balanced state may clearly be noticed.

With a similar highly non-balanced state, we have made a similar computation for the development of a disturbance of $100 \mathrm{~km}$ in size. In this case the development of this disturbance also consists of three stages with three different time scales. The adjustment stage (1st stage) lasts about quarter of an hour. Then it goes to the 2nd stage which lasts to about one hour and finally it enters into quasi-steady state. (Figures neglected)

Further lessening the space scale, we have computed the development of a disturbance of $10 \mathrm{~km}$ in size initially in highly non-balanced state. Its initial conditions are also given by the first set of equation (4), and $f(r, 0)$ also given by figure 1. The results of computation of $V_{r}, V_{\theta}$ and $C$ at different time are given by figure $4 \mathrm{a}$, $4 \mathrm{~b}$ and $4 \mathrm{c}$ respectively. From these figures, it may be seen that in first minute and half the

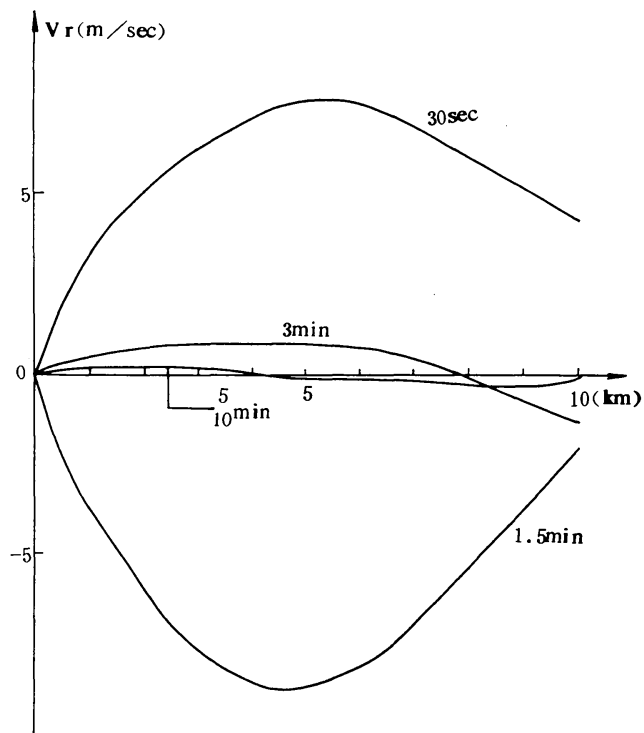

a) The radial distributions of $V_{r}$. fields of $V_{r}, V_{\theta}$ and $C$ undergo violent adjustments; from about one minute and half to about 5 minutes this disturbance further develops but much more slowly than the first stage and finally after about 5 minutes it enters into a quasi-steady

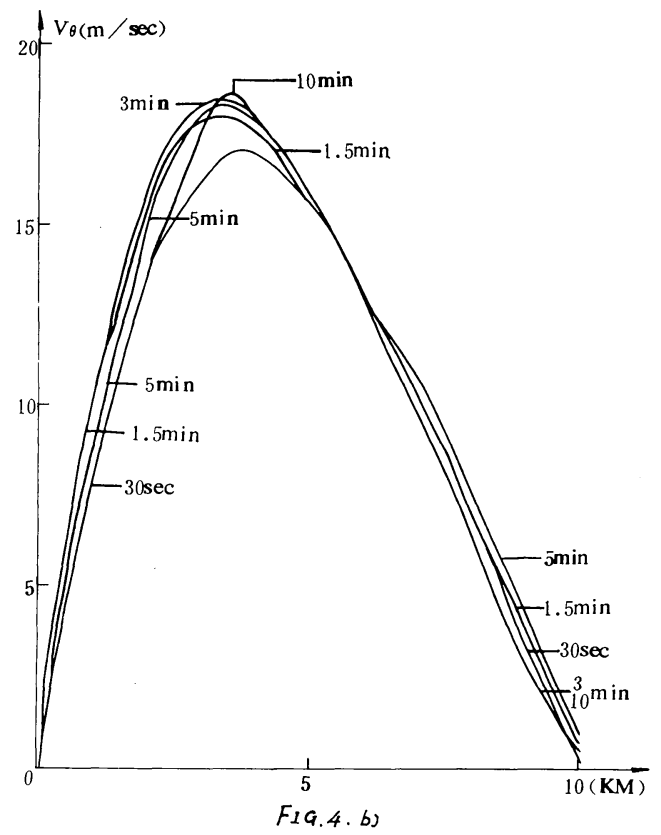

b) The radial distributions of $V_{\theta}$.

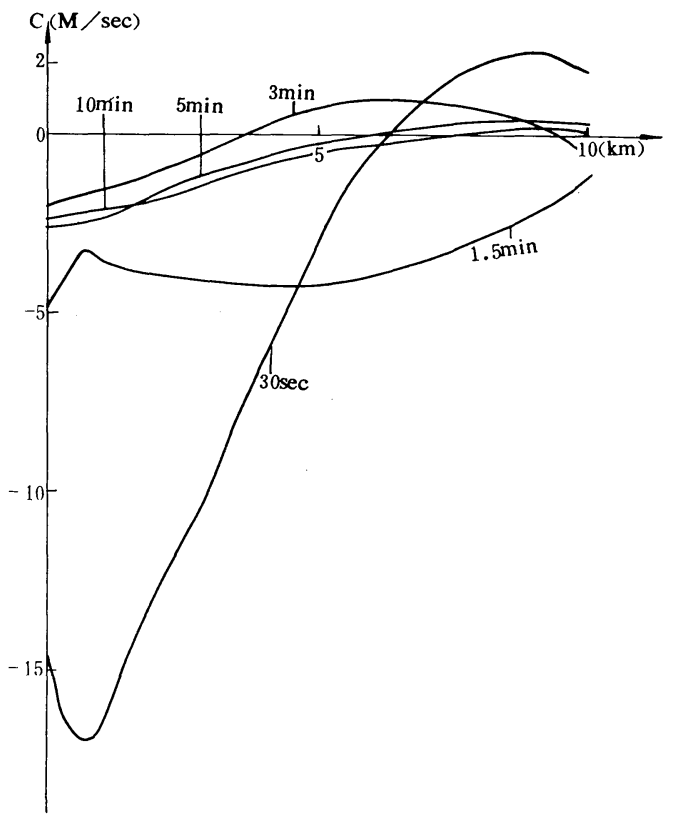

c) The radial distributions of $C$ (deviation from the initial).

Fig. 4. The distribution of $V_{r}, V_{\theta}$ and $C$ of the small scale motion at different stage. 
state.

Numerical computations with initial conditions given by second set of equations of equation (4) were also carried out. Similar results were obtained. We shall not discuss them here.

To conclude this section we may quote a numerical simulation of strong convection due to surface heating by Amirov (1968). His results showed that: "At $t=8$ minutes, the cloud appears at a height of 600 meters and then it rapidly develops. At $t=10$ minutes, the vertical speed at the top of the cloud reaches $300 \mathrm{~m} / \mathrm{min}$. ...... Between $10 \mathrm{~min} . \leq t \leq 20 \mathrm{~min}$., the averaging growing speeds decrease to $120 \mathrm{~m} / \mathrm{min}$. ...... Between 20 minutes $\leq t \leq 40$ minutes, the average growing speed is $20 \mathrm{~m} / \mathrm{min}$.

The numerical computation is carried out up to 120 minutes. But the solution at this time practically does not have any difference from that at 60 minutes. Therefore we consider that the cloud has reached a steady state at $t=60$ minutes."

From this description we may see that Amirov's numerical simulation of the formation of strong convective cumulus cloud also supports our theory that the development of a disturbance consists of three stages with three different time scales.

\section{The conservation of potential vorticity and the space-scale effect}

The above observations and the numerical simulations all show that the development of the weather systems, large or small, in the atmosphere consists of three stages, each stage having its own characteristic time-scale. In this section we shall show that when there is no source or sink, during the development of the weather systems, large or small, there exists a conservation of potential vorticity and a space-scale effect on the process of the development. For the large scale adiabatic motion, the vorticity equation may be written as

$$
\frac{d}{d t}\left[f+\zeta+\frac{\bar{f}}{C^{2}} \frac{\partial}{\partial \xi}\left(\xi^{2} \frac{\partial \phi}{\partial \xi}\right)\right]=0
$$

Here $\xi=P / P_{0}, P_{0}$ being a constant (as $1,000 \mathrm{mb}$ ); $C^{2}=\alpha R T, \alpha=R\left(\Gamma_{\alpha}-\Gamma\right) / g$ and $\Gamma_{\alpha}$ and $\Gamma$ being respectively the dry adiabatic and the observed temperature lapse rate; and $\bar{f}$ is the Coriolis parameter considered as a constant.* This conservation of potential vorticity holds true for

* In deriving (5), $\mathrm{C}^{2}$ is assumed to be independent of p. all the three stages.

To non-dimensionalize the potential vorticity we have

$$
\begin{aligned}
\zeta+f+\frac{\bar{f}}{C^{2}} \frac{\partial}{\partial \xi}\left(\xi^{2} \frac{\partial \varphi}{\partial \xi}\right) \\
=\frac{\bar{\phi}}{L^{2}} \nabla^{2} \psi^{\prime}+\frac{\bar{f}}{\bar{\phi}} \bar{\psi} f^{\prime}+\frac{\bar{f} \bar{\Phi}}{C^{2}} \frac{\partial}{\partial \xi}\left(\xi^{2} \frac{\partial \phi^{\prime}}{\partial \xi}\right) \\
=\frac{\bar{\phi}}{L}\left[\nabla^{2} \phi^{\prime}+\frac{L^{2} \bar{f}^{2}}{\bar{\Phi}} f^{\prime}+\frac{L^{2} \bar{f}^{2}}{C^{2}} \frac{\partial}{\partial \xi}\left(\xi^{2} \frac{\partial \phi^{\prime}}{\partial \xi}\right)\right] \\
=\frac{\bar{\psi}}{L^{2}}\left[\nabla^{2} \phi^{\prime}+\frac{L^{2}}{L_{0}^{2}}\left\{1+\frac{L}{a} y+\frac{\partial}{\partial \xi}\left(\xi^{2} \frac{\partial \phi^{\prime}}{\partial \xi}\right)\right\}\right]
\end{aligned}
$$

Here $L_{0}=C / \bar{f}$ is Rossby's radius of deformation, $\bar{\Phi} \sim C^{2}$ and we have used the relation $\bar{\psi} \sim \bar{\Phi} / \bar{f}$ in the last term. And the quantities with prime are non-dimensional. From this it is seen that when $L<L_{0}$ the wind field governs the development and when $L>L_{0}$ the pressure field governs the development. The effect of space scale on the geostrophic adjustment, i.e., the first stage has already been discussed by quite a few meteorologists, for example, see Yeh (1957), Zeng (1963) and Chen (1963). It is seen here that this effect not only applies to the first stage, but also to all the three stages.

For meso-scale systems, the relative vorticity $\zeta$ neglected against $\bar{f}$ in equation (5) must be retained. This equation becomes then

$$
\frac{d}{d t}\left[\ln (\zeta+f)+\frac{1}{C^{2}} \frac{\partial}{\partial \xi}\left(\xi^{2} \frac{\partial \phi}{\partial \xi}\right)\right]=0
$$

since $f$ may be considered as constant in mesoscale motions. Introducing the characteristic scales we have

$$
\begin{aligned}
& \zeta=\nabla^{2} \psi=\frac{\bar{\psi}}{L^{2}} \nabla^{2} \psi^{\prime}=\frac{\bar{\psi}}{L^{2}} \zeta^{\prime}, \varphi=\bar{\Phi} \varphi^{\prime}, \\
& \Omega=f+\zeta \text { and } \Omega=\bar{\Omega} \Omega^{\prime},
\end{aligned}
$$

here $\bar{\Omega}$ being the scale value of absolute vorticity. Introducing $\bar{\psi} \sim \bar{\Phi} / \bar{\Omega}$ we have

$$
\frac{d}{d t}\left[\ln \Omega^{\prime}+\frac{L^{2}}{L_{0}{ }^{2}} \frac{\partial}{\partial \xi}\left(\xi^{2} \frac{\partial \varphi^{\prime}}{\partial \xi}\right)\right]=0
$$

Now we can see that there is a similar spacescale effect on the process of development of meso-scale systems. But here $L_{0}^{\prime}=C / \bar{\Omega}$ not only depends on the background parameter, $(C$ and $f$ ) but also depends on the characteristics of the motion field $(\bar{\Omega})$.

For small scale motions it is not easy to be treated, for hydrostatic relation can not be used. Let us solve a simple case of two-dimensional $(x, z)$, adiabatic and incompressible motion. The 
vorticity equation for this case may then be the same depth $(H)$ of the weather system, larger written as (Chao and Zhou, 1965; Yeh and $\mathrm{Li}$, thermal action will be needed to initiate this 1980)

$$
\left(\frac{\partial}{\partial t}+u \frac{\partial}{\partial x}+w \frac{\partial}{\partial z}\right) \nabla^{2} \psi=-\frac{g}{\bar{\rho}} \frac{\partial \rho^{*}}{\partial x}
$$

where $\rho^{*}$ is the deviation of density from its mean $\bar{\rho}(z)=\rho(x, z)-\rho^{*}(x, z)$. Write

$$
\begin{aligned}
\frac{\partial \rho^{*}}{\partial x} & =\frac{\partial \rho}{\partial x}=\frac{\partial \rho}{\partial \phi} \frac{\partial \psi}{\partial x}=-\frac{\partial \rho}{\partial \phi} w=-\frac{\partial \rho}{\partial \phi} \frac{d z}{d t} \\
& =-\frac{\partial \rho}{\partial z} \frac{\partial z}{\partial \phi} \frac{d z}{d t}=-\frac{1}{u} \frac{\partial \rho}{\partial z} \frac{d z}{d t} .
\end{aligned}
$$

Neglecting the individual variation of $(1 / u)$ $(\partial \rho / \partial z)$, then $(8)$ can be written as

$$
\left(\frac{\partial}{\partial t}+u \frac{\partial}{\partial x}+w \frac{\partial}{\partial z}\right)\left(\nabla^{2} \psi+\frac{g}{u} \frac{1}{\bar{\rho}} \frac{\partial \rho}{\partial z} z\right)=0
$$

This is the equation of potential vorticity. This equation is similar to Long's (1953) equation which has been widely used in the analysis of small scale motions. But Long's $u$ in the second part of the potential vorticity is the velocity of the environmental basic current. However, here in our case it includes the velocity of the disturbance.

Let $U$ and $H$ be the scale values of horizontal velocity and height. We can write (9) as

$$
\begin{aligned}
& \left(\frac{\partial}{\partial t}+u \frac{\partial}{\partial x}+w \frac{\partial}{\partial z}\right) \\
& \quad \times\left(\zeta_{y}^{\prime}+\frac{H^{2}}{L_{0}^{\prime \prime 2}} z^{\prime} u^{\prime-i} \frac{\partial \rho^{\prime}}{\partial z^{\prime}}\right)=0 .
\end{aligned}
$$

Here we have used $U / H$ as the characteristic value of the vorticity $\zeta y$ and

$$
L_{0}{ }^{\prime \prime}=u^{2} / \frac{g}{\bar{\rho}}\left|\frac{d \bar{\rho}}{d z}\right| .
$$

From the above equation we can deduce similar conclusions about the influence of scale on the development of small-scale motion. The first part of equation (10) may be considered as the dynamically produced vorticity and second part as the thermally produced vorticity. For deep systems $\left(H \gg L_{0}{ }^{\prime \prime}\right)$ the thermal action will govern the process of the development. For shallow systems $\left(H<L_{0}{ }^{\prime \prime}\right)$ the dynamic action will be the predominant factor. We may also see that $L_{0}{ }^{\prime \prime}$ is inversely proportional to $(g / \bar{\rho})$ $(\partial \cdot \rho / \partial z)$ (square of Brunt-Väisälä frequency). The larger the value of $(g / \bar{\rho})(\partial \bar{\rho} / \partial z)$, i.e., the more stable the stratification, the smaller the value of $L_{0}{ }^{\prime \prime}$, then the more predominant the thermal influence will be. In other words, for system in more stable atmosphere than in less stable atmosphere. Further $L_{0}^{\prime \prime}$ is directly proportional to the scale velocity $U$. Therefore for the systems of equal depth, the dynamic factor is more predominant in the field of larger velocity than in the field of smaller velocity.

\section{The adaptation between the stream field and the pressure field and the adaptation between the stream field and the potential vorticity field}

Using a linear system Oboukhov (1949) showed that for large-scale motions during the stage of geostrophic adjustment the potential vorticity remains constant with time. Later $\mathrm{Yeh}$ and $\mathrm{Li}$ (1965) demonstrated that for highly non-geostrophic motions the non-linear terms are much smaller than the linear terms and the more nongeostrophic the motions are, the much more smaller the non-linear terms are. Therefore for the first stage, the geostrophic adjustment stage, of a large-scale motion, the potential vorticity equation may be written as

$$
\frac{\partial Q}{\partial t}=0 \quad \text { or } \quad Q=Q_{0} .
$$

$Q$ being the potential vorticity and $Q_{0}$ being its initial value. Entering into the second stage of the development, the quasi-geostrophic stage, of a large-scale motion, the non-linear terms should be retained. The potential vorticity equation, therefore, should be written as

$$
\frac{\partial Q}{\partial t}=J(\phi, Q)=0
$$

where $J$ is the Jacobian operator. In the third stage the motion is quasi-steady and thus the potential vorticity equation can be written as

$$
J(\phi, Q)=0
$$

From the above we may see that in the life of development of a large-scale weather system it consists of three stages. In the first stage the motion is highly non-geostrophic and the development in this stage leads the motion to quasigeostrophy (the geostrophic adjustment). From equations (11b) and (11c) we may also see that from the second stage on the motion enters into an another physical process, i.e., the process of adaptation between the stream line field and potential vorticity field. In this process these two fields so adjust with each other that the angle between the stream lines and the lines of 
equal potential vorticity becomes smaller and smaller. As the angle approaches nil the largescale weather system attains its mature stage, further development stops and this weather system enters into the quasi-steady state. Li (1979) called this process the potential vorticity adaptation of large-scale motions.

For the motions the space scale of which is small enough so that $f$ can be considered as constant or even ignored, but also large enough so that the $w$-terms in the equations of motion can still be neglected, the non-linear terms are also much smaller than the linear terms when the motion is in highly non-balanced state of forces, i.e., the first stage (Yeh and $\mathrm{Li}, 1965$ ). Recently Yeh and Li (1980) again showed that for very small-scale motions (like cumulus) where the hydrostatic relation does not hold the non-linear terms are also smaller than the linear terms when the motion is highly in non-balanced state of forces. Therefore in first stage development of the meso-scale and cumulus-scale motions the potential vorticity equation may also be written as equation (11a). While in second and third stage the variation of potential vorticity may similarly be described respectively by equation (11b) and (11c). But $Q$ has different forms for different space scales.

Therefore for meso- and small-scale weather systems, there is also certain process of adjustment between the velocity field and pressure field when the motion is in highly non-balanced state of forces and the result of this process will lead the motion to a state of certain quasibalance of forces. Then the non-linear terms become important and the potential vorticity equation (11b) should be applied. From this time on there will be also a process of adjustment between the stream field and potential vorticity field. As for large-scale motions, the angle between the stream lines and the lines of equal potential vorticity will also become smaller and smaller as the development of meso- and small-scale weather systems proceeds. When this angle approaches nil, the weather systems reach the mature stage and enter into the quasi-steady state.

\section{Concluding remarks}

In this paper we have shown that the atmospheric motions, large- or small-scale, proceed usually under quasi-balanced state of forces. When this quasi-balanced state breaks down, it undergoes a very rapid adjustment to bring the motion back to the state of quasi-balanced forces. The time scale of this adjustment is so short that it usually can not be observed in real atmosphere. In this stage of development the non-linear terms are smaller than the linear terms. After this stage the non-linear terms become important and the motion resumes its quasibalanced state of forces. The real development of a weather system which can be observed in the atmosphere actually happens in this stage. The time scale of this second stage is much larger than that of the first. The weather system usually is brought fairly rapidly to its mature state of development in this stage. Then the weather system enters into its quasi-steady state, the third stage, the time scale of which is again much longer than that of the second stage. Due to various reasons a weather system will of course die out. Counting this dying stage, the life of a weather system consists of four stages.

It is also shown in this paper that for adiabatic and frictionless atmospheric motions, large- or small-scale, there exists a potential vorticity equation. For the first, second and third stage of the development, this equation may respectively be reduced to

$$
\begin{aligned}
& \frac{\partial Q}{\partial t}=0 \quad \text { or } \quad Q=Q_{0}, \\
& \frac{\partial Q}{\partial t}+J(\phi, Q)=0 \text { and } J(\psi, Q)=0
\end{aligned}
$$

where $Q$ has different forms for different spacescale atmospheric motions. And for the motion of any space-scale large, meso or small, there exists a critical scale $L_{c}$ which has different forms for different space-scale motions. When a disturbance possessing a scale $L>L_{c}$, the pressure field (or thermal field) will govern the development of the disturbance, while when $L<L_{c}$ the velocity field (or the dynamic field) will govern the development.

From the observations as well as from the numerical simulations it is also seen that the time scale is closely related to the space scale, the larger the space scale, the greater the time scale. Why so? Why can not small-scale weather systems usually live longer than large-scale weather systems? What is the dynamic relation between time scale and space scale? This is an interesting problem worth studying. For largescale motions, the mean meridional temperature gradient determines the average vertical shear of zonal wind $(\partial u / \partial z)$. When $\partial u / \partial z$ reaches certain value, it will lead to long wave instability. 
The wave length of most intense instability gives the space scale of large-scale motion and the $e$-folding time of the amplitude of the most unstable wave determines the time scale (of second stage) of the large-scale motion. Similarly for small-scale motions there will also be certain kind of instability. The wave length of most unstable wave and the $e$-folding time of the increase of its amplitude will determine respectively the space scale and the time scale (of second stage) of the small-scale motion. Could the processes described here give the dynamic relation between the space scale and time scale?

We also know that in the dynamics of the atmosphere, besides the time scale and space scale there is another fundamental scale, the velocity scale. What then determine the velocity scale of various kinds of atmospheric motions? What is the dynamic relation between the velocity scale and the other two scales? This is the another problem worth studying.

\section{References}

Amirov, A. D., 1968: Influence of an artificial source of heat on the cumulus evolution. Numerical experiment. Izvestiya of Academy of Science USSR Atmospheric and Oceanic Physics Vol. IV, 152-159.

Chen, Chu-shih, 1963: On the formation and the destruction of the thermal wind in a simple baroclinic atmosphere. Acta Meteorologica Sinica. V.
33, 153-161.

Chao, Jih-ping and R. S. Wu, 1980: Characteristics of multi-time scale of motion and temporal boundary layers in rotating atmosphere. Scientia Atmospherica Sinica V. 4, 138-147.

Li, M. T. 1979: On the stages of large scale motion of the atmosphere. Scientia Sinica 1979. 6, 599-607.

Long, R. R. 1953: Some aspects of the flow of the stratified fluid. Tellus, 5, 42-56.

Oboukhov, A. M. 1949: The problem of the geostrophic adaptation. Izvestiya of Academy of Science USSR Series Geography and Geophysics, Vol. 13, 281-289.

Yeh, T.C. 1957: On the formation of quasi-geostrophic motion in the atmosphere. J. Met. Soc. Japan. The 75th Anniversary Volume. 130-134.

Yeh, T.C. and Li, M. T. 1965: On the adaptation of the atmospheric motion. Science Press, Peking. 1965: On the adaptation between the wind field and pressure field in small and mesoscale motion in the atmospheric motion. Scientia Sicina V. XIV N. 3, 437-450.

— 1980: On the multi-time scales of different kind motion in the atmosphere. Proceedings of second conference of numerical forecasting, Peking, 22-28, December 1977.

Zeng Qing-cun, 1963: The influence of disturbance on the process of adaptation and the problems of application of the observed wind fields. Acta Meteorologica Sinica. 33, 37-50.

, 1963: The adjustment and evolutional process in the atmosphere. Acta Meteorologica Sicina. 33, 163-174, 281-289.

\title{
大気運動のスケール特性について
}

\author{
Tu-cheng Yeh (叶篤正) $\cdot$ Mai-tsun Li
}

(中国科学院・大気物理研究所)

\begin{abstract}
大気中の天気系の大部分は, 大規模でも小規模でも，3つの発展段階から成っていることを示す。すなわち， 調節段階 (大規模運動に対しては地衡風調節と呼ばれる), 発達段階, そして準定常段階である。この 3 つの発 展段階に対応して $3 つ の$ 時間スケールがある。調節段階の時間スケールは最も短かく，発達段階はそれよりも長 く, 準定常段階は最も長い。これらの発展段階の物理過程はポテンシャル渦度の場の変遷発展に関係づけられる。 大規模運動に打いて $L_{9}=c / f$ を特性的長さとするスケール効果があるのに類似してメソスケールの運動に対 しては $L_{0}{ }^{\prime}=c / \Omega$ という特性的長さが存在することを示す。ここに $c$ は重力波の速さ， $f$ はコリオリ・パラメ 一ター, $\Omega$ は風速場のもつ絶対渦度の代表值である。或る適当な仮定の下で, 積雲スケールの運動に対しても $L_{0}{ }^{\prime \prime}=\left[u^{2} \frac{g}{\rho} \frac{\partial \rho}{\partial z}\right]^{1 / 2}$ といら特性的長さが存在する。ただし， $u$ と $\rho$ とは風速と密度の代表的な值である。メり スケールと積雲スケールの運動にお活る $L_{0}{ }^{\prime}$ と $L_{0}{ }^{\prime \prime}$ の役割は大規模運動に拈ける $L_{0}$ の役割に類似している。
\end{abstract}

